\title{
Morphological Analysis for Temporary Behaviour Description of Photoelasticity Images on Plastic Films
}

\author{
Juan Carlos Briñez De León ${ }^{1}$, Alejandro Restrepo-Martínez², \\ and Francisco López Giraldo ${ }^{3}$ \\ ${ }^{1}$ M.Sc(c) Automatización y Control Industrial, Grupo de Investigación en Automática, \\ Electrónica y ciencias de la computación, ITM, Colombia \\ juanbrinez@gmail.com \\ ${ }^{2}$ Ph.D en Ingeniería de Sistemas, Grupo de Investigación en Automática, Electrónica y ciencias \\ de la computación, ITM, Colombia \\ alejandromartinez@itm.edu.co \\ ${ }^{3}$ Ph.D en Física, Grupo de Investigación en Automática, Electrónica y ciencias de la \\ computación, ITM, Colombia \\ franciscolopez@itm.edu.co
}

\begin{abstract}
Thermo-deformable plastic films experience an optical birefringence phenomenon when they are subjected to mechanical strain, this phenomenon can be described by color fringes behavior observed in sequences of images acquired through photoelasticity assemblies. This paper proposes a temporary morphological analysis that color fringes experience when the plastic film is deformed. For the image analysis, stereology and erosion surfaces techniques are applied. It achieves the relationship between morphological description and mechanical behavior of the plastic films deformation process. The stereology and erosion surfaces allowed the identification of the appearance of color fringes that gradually increase in size into the image sequence. The morphological results showed that temporary behavior of color fringes could be used for modeling the mechanical behavior of the plastic films deformation process.
\end{abstract}

Keywords: Plastic films deformation, fringe colors, erosion, stereology, photoelasticity.

\section{Introduction}

Plastic films deformation is a common process in food industries to produce packages. These packages have a multilayer structure according to conservation requirements of food products. During the process of making packages, plastic film undergoes extreme changes in temperature and mechanical pressure. Due to those changes, each structure that conforms the multilayer film suffers alterations that could modify its capacity to preserve nutritional and organoleptic qualities of the food product such as allowing passage of gases from outside to the inside of the package and vice versa [1].

The mechanical behavior that plastic films suffer use to be described by techniques that require touching the packages, which could result in losing the material. With the 
aim of reducing losses in the production process, optical properties of the material that are modified by those mechanical changes can be measured systematically to analyze mechanical behavior without touching the material.

When plastic films are exposed to mechanical deformations they undergo an optical phenomenon of birefringence, which allows the capture of color fringes images through polarizing optical assemblies used in photoelasticity studies [3]. Thickness variations during film deformation are related to refraction index changes, which in turn make changes on color fringes during the process (1).

$$
\delta=h\left(n_{1}-n_{2}\right)
$$

Where " $\delta$ " is phase difference, " $h$ " is sample thickness and " $n_{1}$ " and " $n_{2}$ " are the refraction index of material. Given that expression (1) changes during the plastic film deformation process and due to the conventional photo-elasticity, studies require analysis of constant thickness models through multiple captured images to make different configurations of polarization in a specific observed point. We can say that the mechanical behavior description of the plastic films deformation process using photoelasticity tools is complex.

In this work, structural changes that color fringes on images captured during the deformation process are used to describe the mechanical behavior process. To do this, morphological analysis for image processing is implemented. Image acquisition is made through optical assembly of polarization proposed in photoelasticity studies $[2,3]$.

Morphological analysis of image fringes is implemented through two methods. The first one uses stereology graphics to obtain a temporal representation of the fringes size changes, taking into account percentage changes on images areas. The second one uses a graphic representation of a temporal morphologic surface created from erosion cycles in each analyzed image.

The assembly for image acquisition is implemented around the plastic film deformation assembly and this process will be described. Then, implemented techniques for morphological analysis of fringes behavior will be described in detail. Finally the results and conclusions of the work will be presented.

\section{Approach}

\subsection{Polarizing Optical Assembly for Image Acquisition}

Due to thermodeformable plastic films suffering birefringence when they are under mechanical stress, fringes colors are observed over the material surface when it is disposed in a polarization optical assembly implemented around the traction machine.

Although photoelasticity studies uses several polarization assemblies to analyze the fringe patterns, in this work a plane polariscope was used because it includes fewer 
optical elements, facilitating the assembly configuration. An LCD screen was used to illuminate the sample [2].

The rotation angle between the polarization axes changes the light intensity emerging from the optical assembly, as shown in (2).

$$
I=I_{m}\left[1-\operatorname{sen}^{2}(2 \theta) \operatorname{sen}^{2}(a \delta)\right]
$$

Where ' $I_{m}$ ' represents the maximum intensity transmitted by the light source, ' $\theta$ ' is the rotation angle between the polarizations axis, and ' $a \delta$ ' is a phase delay function. In this work, the rotation angle is 90 degrees, which allows obtaining the greatest intensity on the optical assembly [3]. Figure 1 shows the optical assembly scheme implemented around the deformation assembly.

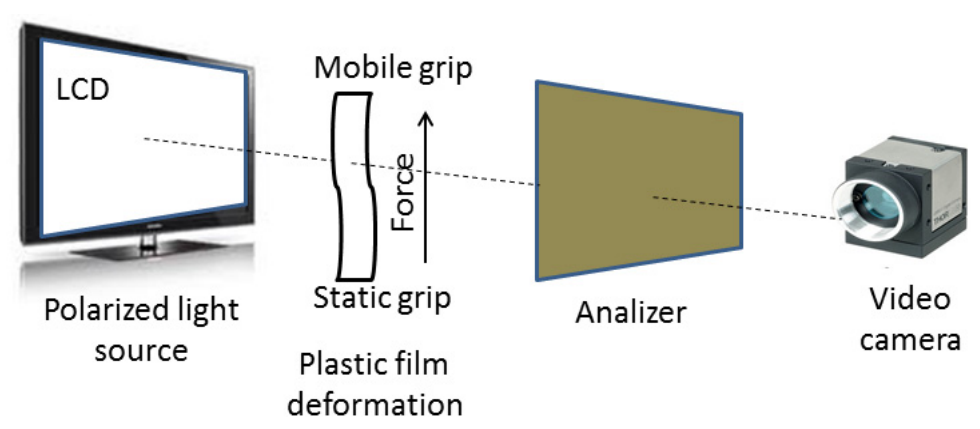

Fig. 1. Schematic model of optical assembly based on a plane polariscope assembly

Color fringes are recorded in a 30fps velocity video using a Thorlabs DCU224C camera. The video caught is approximately 5 minutes and is divided into a 4500 -color image sequence that contains the fringes temporary behavior during the process, which is expressed as a number of obtained images.

Images in the sequence have a size of $1280 \times 1024$ pixels. After relevant information of each image has been taken, new images of 576×150 pixel size are generated.

The traction machine allows the measurement of stress required for the plastic material deformation. Sampling rate of these dates will be adjusted to the cameras capturing velocity.

Relevant image information extracted from sequences is presented in the Fig. 2, in which formation, growth and movement of color fringes can be observed. Fringes on image sequences move from the static grip zone to the mobile grip zone. The linkage between the sampling time of the image and its number inside the sequence is presented in the figure. In this paper the figures in function to the number of images are presented. Also showing a comparison between color images and binarized images for the R channel. 


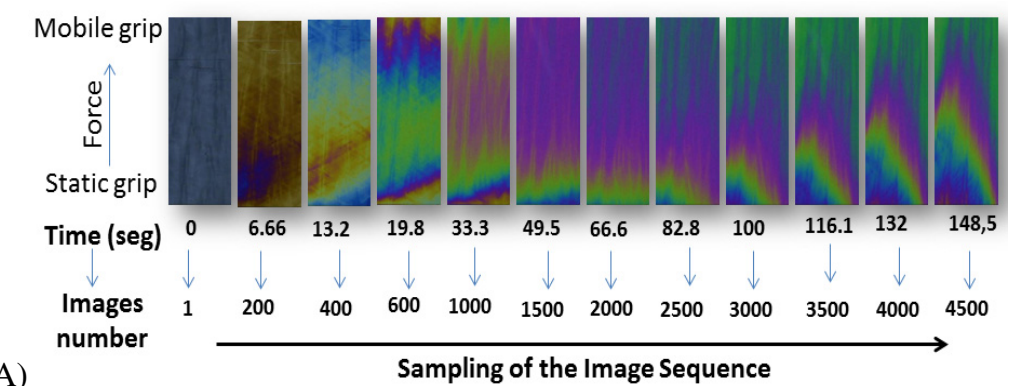

A)

Sampling of the Image Sequence

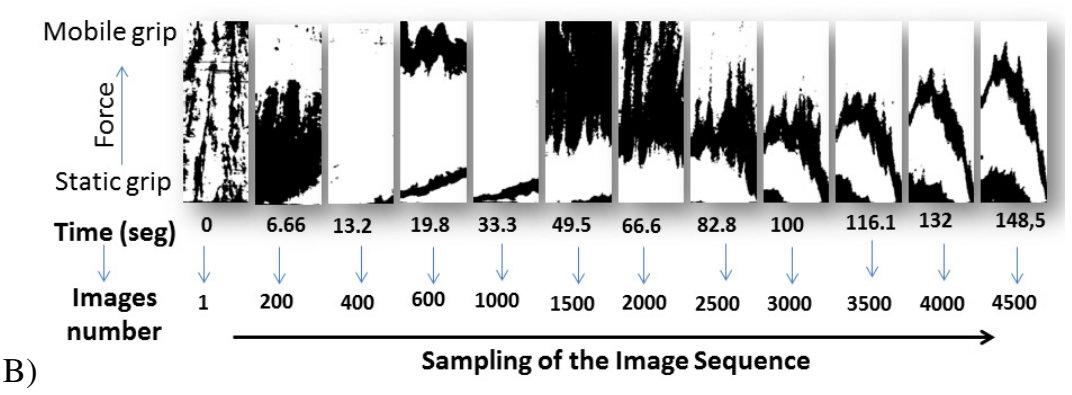

Fig. 2. Images extracted from the image sequence acquired during the plastic film deformation. A) Color image. B) Binarized image Plastic film deformation.

\subsection{Plastic Film Deformation}

Different to conventional techniques used in photoelasticity studies with constant thickness samples supporting fixed load [4], in this work, plastic film is deformed by mechanic traction using a universal Shimadzu AGX 100KNewton machine. Plastic film deformation is made by upper-grip displacement, as described in [2].

Deformation test parameters and plastic film dimensions were established according to D882-10 rule, which applies to plastic films deformation with thickness lower than one millimeter [5]. A thermodeformable multilayer plastic film of $130 \mu \mathrm{m}$ thickness was used for the deformation test. Plastic film dimensions are $2.5 \mathrm{~cm}$ wide and 15 $\mathrm{cm}$ long. Deformation velocity is $25 \mathrm{~mm}$ per minute.

The mechanical behavior of plastic film deformation exhibits a characteristic curve, in which the stress is variable during the process, as described in [1]. The area of the graphic that undergoes the fastest stress changes is identified as the elastoplastic zone, and the zone that experiences gradual stress changes is denominated viscoelastic zone.

\subsection{Stereology for Morphology Description}

Stereology is normally used for 3D microstructure measurements, starting with dividing the object in sections of plans. The volume measurement is achieved through the fraction areas, the bi-dimensional surfaces are described as lines and dimensional 
curves are described as points [7]. A plane section of a 3D object can be compared with a cut or bi-dimensional image taken from the object.

Although most of stereological measurements are made to obtain a representative measurement of 3D microstructures using plane sections taken uniformly through the sample, the technique can also be used to estimate geometrical properties of objects. In this work the stereology is used to describe the behavior of size changes that fringes experience during the deformation process.

For the temporary behavior description of the changes in the fringes fractions " $L_{L}$ " inside the binarized image, a grid is intersected using the logical operation OR with each one of the sequence images. The grid used is a logical image with the same dimensions of the analyzed image created with black lines put on a white background. The expression for the fringe fraction is described in (3).

$$
L_{L}=\frac{\text { Intersected pixels }}{\text { Grid pixels }} * 100 \%
$$

In expression (2) the division's result is multiplied by 100 to express the value in percentage [7]. Each photo-elasticity image of the acquired sequence during the plastic film deformation is analyzed for each RGB color channel. Each result for the fringes fraction calculation is kept in an organized way inside a data vector. The amount of data vectors is the same as the amount of sequence images, averaging around 4350. A fringe fraction with $100 \%$ indicates that the entire analyzed image contains homogeneous fringes around its entire surface.

\subsection{Eroded Surfaces for Morphology Description}

Although granulometry is a common tool used for the morphological descriptions of geometrical structures, the application of traditional granulometry techniques does not allow fringes descriptions because the opened cycles do not generate significant reductions in the fringes sizes. However it is possible to make a morphological fringe description by replacing opened cycles by erosion cycles [8].

The morphological analysis using the erosion cycles on the binarized photoelasticity images is described step by step, starting with the binarized images of photoelasticity for each RGB color channel.

Each image of sequence "I" is binarized by each RGB color channel, where "Ar" is the binarized image of the "R" channel, "Ag" is for channel "G" and "Ab" for channel "B". In this work the erosion cycle's equations are presented from channel "R" because the procedure is the same for the " $G$ " channel and the " $B$ " channel. The binarized image "Ar" can be represented by (4).

$$
\boldsymbol{A r}=\left[a_{i, j}\right]_{m \times n}
$$

Where "m" and " $n$ " are the image dimensions, "Ar" is the erosion using a structuring element, " $\mathrm{H}$ " is the type disc with a radius of 10 pixels, which creates a "Br" image through (5) and represented through (6). 


$$
\begin{aligned}
& \boldsymbol{B} \boldsymbol{r}=\boldsymbol{A r} \boldsymbol{H} \\
& \boldsymbol{B} \boldsymbol{r}=\left[b_{i, j}\right]_{m x n}
\end{aligned}
$$

"Br" is eroded 50 consecutive cycles, which are sufficient to achieve the reduction of the fringes in the image. The result of each erosion cycle creates an image that is stored in "Cr", as shown in (7).

$$
\boldsymbol{C r}=f((\boldsymbol{B} \boldsymbol{r} \boldsymbol{H}), k)
$$

Where " $k$ " represents the number of erosions, "Cr" can be represented by (8), taking " $\mathrm{m}$ " and " $\mathrm{n}$ " as the image dimensions and " $\mathrm{T}$ " as the erosions number.

$$
\boldsymbol{C r}=\left[c_{i, j, k}\right]_{m \times n x T}
$$

The second step of the technique is to count the pixels of the image resulting by subtracting "Ar" with "Cr", the pixels resulting from each erosion cycle are stored in a "Pr" position, as shown in (9).

$$
\boldsymbol{P r}=\left[p_{k}\right]_{T}
$$

Where,

$$
p_{k}=\sum_{i}^{m} \sum_{j}^{n}\left(\boldsymbol{A} \boldsymbol{r}_{i, j}-\boldsymbol{C} \boldsymbol{r}_{i, j, k}\right)
$$

When the sequence is evaluated it creates a vector "Pr" for each image, which is stored in the "Sr" array, as shown in (11).

$$
\boldsymbol{S r}=\left[s_{k, l}\right]_{T \times N}
$$

Where " $k$ " represents the number of cycles and " $l$ " is the number of frames temporarily extracted from the deformation video. Each "Sr" element can be presented as:

$$
s_{k, l}=f(\boldsymbol{P r}, l)
$$

The erosion cycles results are presented when "Sr" is graphed through the 3D surface

\subsection{Relationship between the Morphological Behavior and the Behavior of the Forces in the Deformation Process}

It is possible to compare mechanical behavior and morphologic temporal behavior of fringes using linear correlation because force behavior data is synchronously sampled with photo-elasticity images captured during the deformation process.

To describe linearity between mechanical behavior of deformation and temporal behavior of color fringes, Pearson correlation coefficient is applied. Before applying it data is normalized. Correlation coefficient ranges between "- 1 " and " 1 ", where "-1" is 
an inverse correlation, " 1 " is a direct correlation and " 0 " does not have any correlation. To calculate the correlation coefficient the expression shown in (8) was used.

$$
r=\frac{\sum_{I}\left(A_{1}(I)-\overline{A_{1}}\right)\left(A_{2}(I)-\overline{A_{2}}\right)}{\sqrt{\left(\sum_{I}\left(A_{1}(I)-\overline{A_{1}}\right)^{2}\right)\left(\sum_{I}\left(A_{2}(I)-\overline{A_{2}}\right)^{2}\right)}}
$$

Where " $A_{1}$ " and " $A_{2}$ " are the data vectors to be compared, " $\overline{\mathrm{A}_{1}}$ " and " $\overline{\mathrm{A}_{2}}$ " are the averages of vectors and " $r$ " is the correlation coefficient value.

\section{$3 \quad$ Results and Analysis}

\subsection{Mechanical Behavior of Deformation}

Fig. 3 shows temporal behavior of applied forces during the deformation process. This figure also shows the elastoplastic and viscoelastic zones. The horizontal axis of the graph ("images number") is data sampling, which depends on number of sequence images.

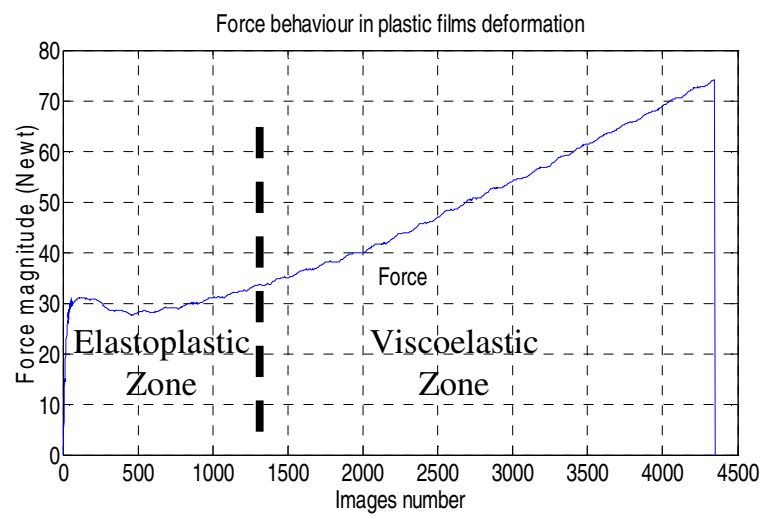

Fig. 3. Force behavior as a function of the number of images recorded in plastic film deformation

The transition between the elastoplastic and the viscoelastic zones in the mechanical behavior of deformation process is noted by taking into account the behavior of the force, in other words, when the data passes from rapid to gradual changes.

\subsection{Stereology in Fringes Description}

In stereology, high fringe percentages imply images with bigger size fringes and low percentages imply images with smaller size fringes. Variations in fringe percentages shows that in the beginning of the deformation process there is a zone where fringe sizes oscillate, the so-called "fast changes zone". There is another zone where fringes undergo gradual changes, the so-called "slow changes zone"; as shown in fig.4 


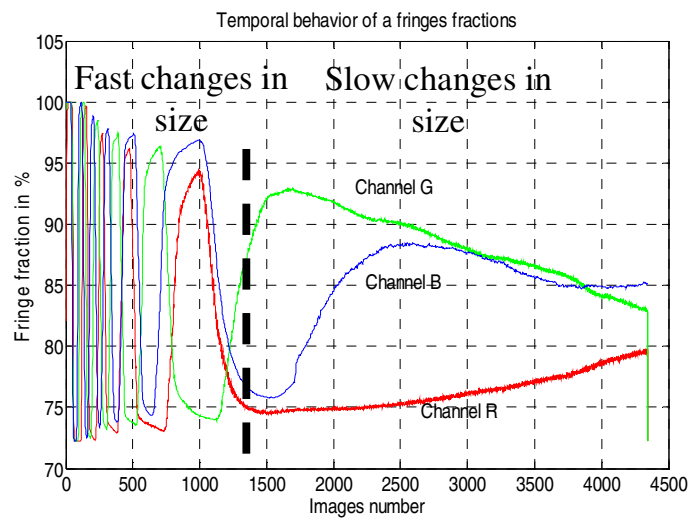

Fig. 4. Temporal behavior of fringe fractions as a function of the number of sequence images

The transition point between fast change zones and low change zones is given by using the stereology vectors derived function, as shown in [2].

Fast change zones coincide with zones where deformation presents elastoplastic behavior. Low change zones coincide with zones where deformation presents viscoelastic behavior.

\subsection{Eroded Surfaces in Fringes Description}

Behavior of erosion cycles is presented through 3D surfaces, which describe the temporal morphologic behavior experienced by fringes during deformation process. Fig. 5 shows the surface of erosion cycles " $S 3$ " and expresses the number of eliminated pixels for each cycle depending on the number of sequence images. "S3" surfaces are shown from the back view.

A)

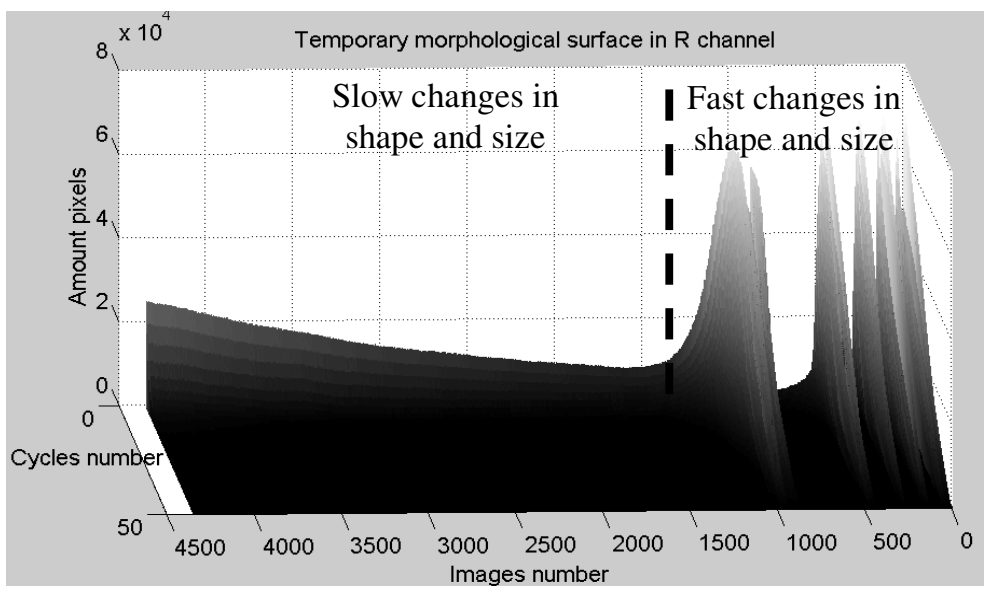

Fig. 5. Temporary morphological surfaces. A) R channel, B) G channel, C) B channel 
B)
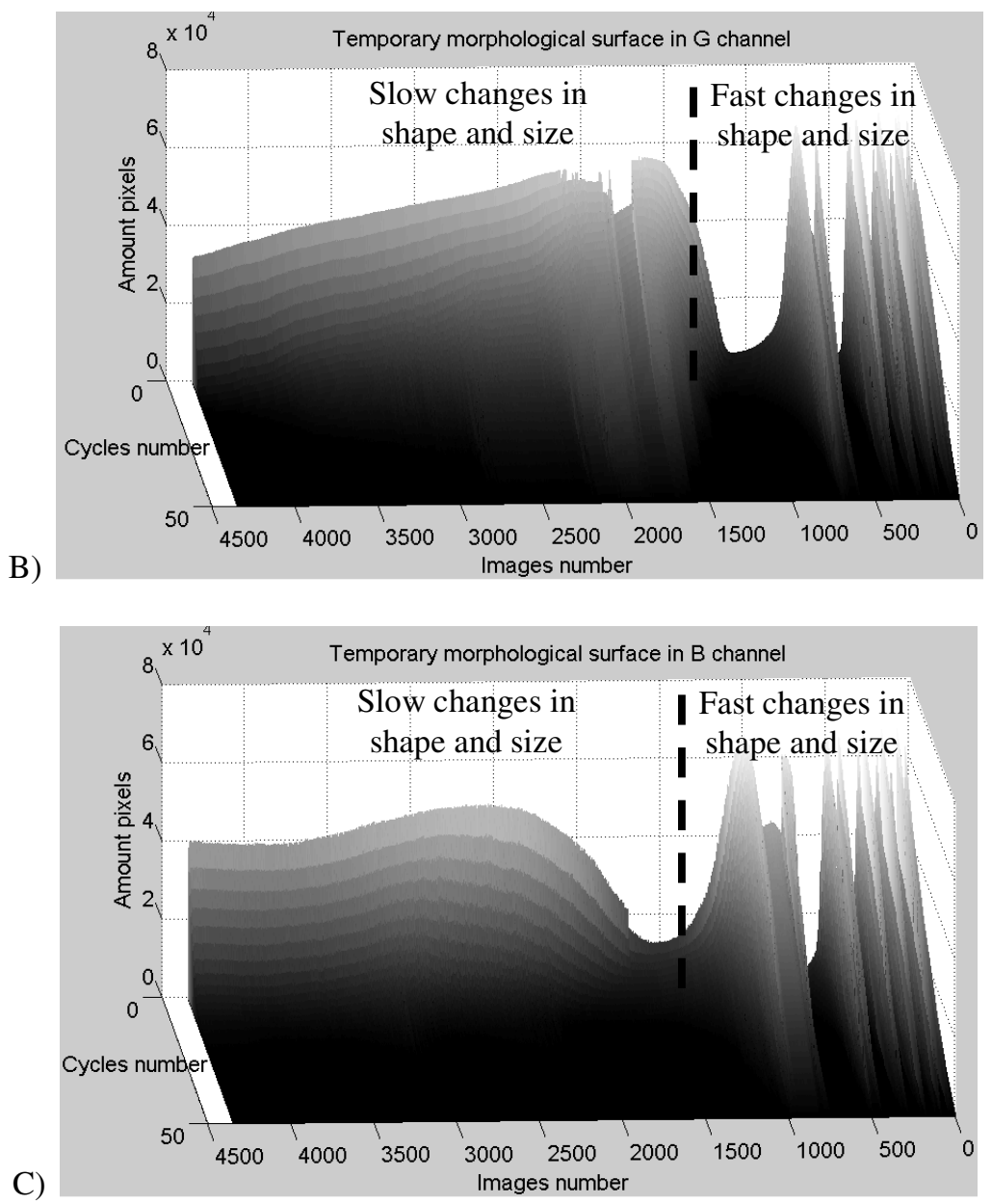

Fig. 5. (continued)

In surfaces of erosion cycles, the eliminated pixels are related with fringes size, it means that a high quantity of pixels implies bigger fringes, and a low quantity of pixels implies a smaller fringe size.

The quantity of needed erosion cycles to remove all pixels from an image increases with fringe size. There is a zone in the beginning of the image sequence, where eliminated pixels have an oscillatory behavior; this zone is the so-called "fast changes zone". The zone where eliminated pixels per cycle change gradually is the so-called "slow changes zone".

According to the application of the derivative function in the first erosion cycle, a dotted line shows a transition between the fast changes zone and the slow changes zone was located, as shown in [2]. 


\subsection{Comparison between Mechanical Behaviour and the Image Analysis}

Graphics analysis allows to identify dynamic behavior zones (mechanical behavior, stereology and erosion surfaces) during the deformation process, and linear correlation among them allows to associate such behaviors.

Data comparison of mechanical and morphologic behavior is made in three steps: First the obtained results for the whole image sequences are correlated, second, the fast changes zone's data is correlated and third, the slow changes zone's data is correlated. Surface data is correlated only with first erosion cycle. Table 1 has channels RGB correlations for stereology and erosion cycles. Table results show that channel "R" has more correlation for the viscoelastic zone, cannel "G" has an inverse correlation and channel "B" has a low correlation. The other two analyzed steps show low correlation for each channel.

Table 1. Correlation coefficient between mechanical and morphological behavior

\begin{tabular}{lcccccc}
\hline \multicolumn{2}{c}{ Correlation coefficient } & \multicolumn{2}{c}{ between } & \multicolumn{3}{c}{ mechanical and morphological behavior } \\
\hline Zone & \multicolumn{2}{c}{ Stereology - Force } & \multicolumn{3}{c}{ Erosion cycles - Force } \\
All data & $\mathrm{R}$ & $\mathrm{G}$ & $\mathrm{B}$ & $\mathrm{R}$ & $\mathrm{G}$ & $\mathrm{B}$ \\
Elastoplastic & -0.1821 & 0.0868 & 0.0699 & -0.1634 & 0.0613 & 0.0573 \\
Viscoelastic & 0.9746 & -0.2442 & -0.2585 & -0.1812 & -0.2588 & -0.2433 \\
\hline
\end{tabular}

Stereology results are correlated with each erosion cycle of the surface and obtain a data vector for each color RGB channel, which have correlation results for each surface cycle. Fig.6 shows the correlation between stereology and surface of erosion cycles.

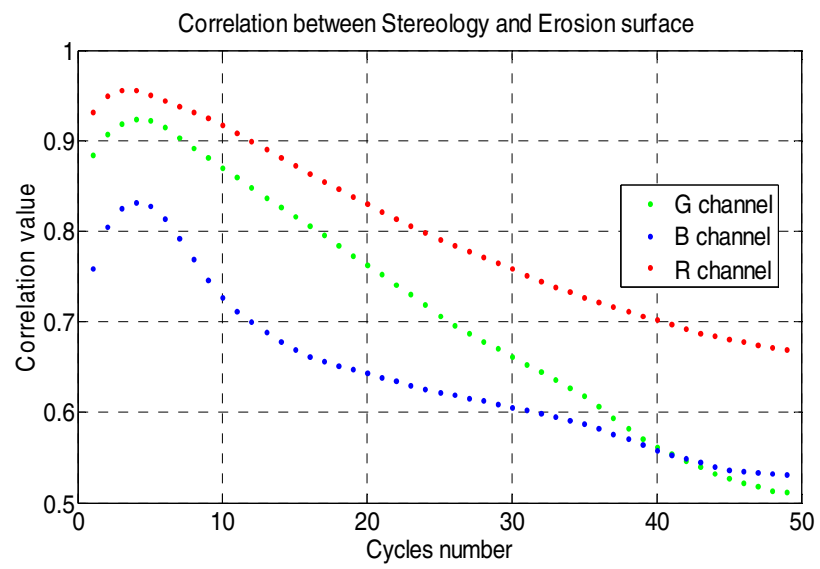

Fig. 6. Correlation coefficient between Stereology vector and Erosion surface cycles for each RGB color channel 
When comparing correlation results from each RGB color cannel, $\mathrm{R}$ has a higher correlation; it means that it is the fringe that best describes fringes morphology. Fig. 6 also shows that the first 5 erosion cycles are which best describe fringes behavior. Then this cycle quantity decreases.

\section{Conclusion}

Photo-elasticity image analysis through stereology and erosion surfaces in plastic films deformation process shows that color fringes change temporally of form and size. In the beginning of the process, fast size oscillations are observed, then gradual size changes are observed.

Stereology and erosion surfaces, applied to image analysis, allows the description of mechanical behavior suffered by a material when it supports a load. Color channels in image analysis show zones that could be associated with viscoelastic zones of mechanical deformation.

Although stereology graphics and surface of erosion cycles allows the analysis of the deformation of mechanical behavior, in comparison with stereology, erosion requires more implementation.

\section{References}

1. Sallem-Idrissi, N., Miri, V., Marin, A., Seguela, R., Lefebvre, J.-M., Krawczak, P., Lacrampe, M.-F.: The role of strain-induced structural changes on the mechanical behavior of PA6/PE multilayer films under uniaxial drawing. Polymer 53, 5336-5346 (2012)

2. Briñez, J., Restrepo, A., López, F.: Métricas de Similitud Aplicadas para Análisis de Imágenes de Fotoelasticidad. Dyna 79, 42-50 (2013)

3. Ramesh, K., Mangal, S.K.: Data Acquisition Techniques in Digital Photoelasticity, vol. 30(1), pp. 53-75. Elsevier (2010)

4. Kale, S., Ramesh, K.: Advancing front scanning approach for three-fringe photoelasticity. Optics and Lasers in Engineering 51, 592-599 (2013)

5. D882-10. Standard Test Method forTensile Properties of Thin Plastic Sheeting. ASTM (March 28, 2011)

6. Gonzales, R., Woods, R.: Digital image processing, 2nd edn., pp. 519-566. Prentice Hall (2001) ISBN: 0-201-18075-8

7. Russ, J., Dehoff, R.: Practical Stereology, 2nd edn., pp. 1-23. Kluwer Academic (2000) ISBN 0-306-46476-4

8. Devaux, M., Brigitte, D., Fabienne, M.: Macro-vision and grey level granulometry for quantification of tomato pericarp structure. Postharvest Biology and Technology 47, 199-209 (2008) 